\title{
Information theory based symmetry classifications of more or less 2D periodic patterns in Islamic building ornaments and Hans Hinterreiter's graphic art
}

\author{
Peter Moeck \\ Portland State University, PORTLAND, Oregon, USA; \\ pmoeck@pdx.edu
}

Possibly for recreational purposes, the very well-established crystallographer Emil Makovicky has for the last 30 to 40 years turned his formidable analytical skills to the classification of the symmetries that underlie patterns of Islamic building ornaments and Hans Hinterreiter's graphical art. These patterns can be characterized as being more or less periodic in two dimensions (2D), see e.g. [1-5]. He stated in [2] that "in performing the symmetrological analysis, we should stay on the same level of accuracy on which the creator of the pattern worked". He had to concede, however, more than 30 years ago that this "certainly brings a certain subjectivity into the process: we should not shun away from it because such a degree of abstraction from imperfections of certain degrees and kinds underlies the entire practice of natural sciences wherefrom the science of symmetry originated" [2]. Utilizing recently developed information theory-based approaches to crystallographic symmetry classifications in 2D [6,7], one can now replace that subjectivity with the objectivity that comes from calculations that involve all pixel intensity values of digital images of such patterns.

These information theory-based approaches to crystallographic symmetry classifications utilize geometric Akaike Information Criteria (G-AICs), which are in essence first-order geometric bias corrected sums of squared residuals between the raw image data and symmetrized versions of this data. G-AIC value ratios are used for the selection of symmetry models to represent the raw data, whereby the need to estimate the level of the "generalized noise" is removed by algebraic means whenever non-disjoint models are involved. Performing the symmetry model selection in reciprocal/Fourier space and basing it exclusively on the structure-bearing complex-valued Fourier coefficients of the image intensity have the advantage of suppressing much of the generalized noise just by calculating the discrete Fourier transform, which is the first step of translation averaging. Symmetrizing the Fourier coefficients and transforming them back into direct space is equivalent to averaging over the asymmetric units.

A simple definition of generalized noise is that it sums up all variations of the intensities of all individual image pixels that are left unexplained by a correct plane symmetry classification. This type of noise is the sum total of all effects of the recording and processing of the digital image and also includes all disturbing effects of "structural defects" in the underlying patterns. For the methods to work (at their current state of development), this kind of noise needs to be considered as approximately Gaussian distributed. Given that there are many sources of that kind of noise with different distributions and that the contribution of none of these sources dominates, this assumption is justified by the central limit theorem.

The new methods allow for objective, i.e. researcher independent, classifications of the full range of crystallographic symmetries, i.e. Bravais lattice type, Laue class, and plane symmetry group, of "noisy" real-world patterns. The identification of the plane symmetry group that can with the highest likelihood, i.e. minimized expected Kullback-Leibler information loss [6,7], be assigned to a noisy digital 2D periodic image of that pattern by an information theory based method enables the most meaningful crystallographic averaging in the spatial frequency domain.

Considering that it is fundamentally unsound to assign an abstract mathematical concept such as a single symmetry type, class, or group with $100 \%$ certainty to a piece of art or the work of artisans, the information theory-based approaches to crystallographic symmetry classifications deliver probabilistic (rather than definitive) classifications. This means that numerically derived confidence levels of the classifications within individual symmetry hierarchy branches are provided with each classification result so that the researcher is presented with objectively derived information, which may be used at the researcher's discretion.

The paper demonstrates the objective classification of a few Islamic building ornaments and examples of Hans Hinterreiter's graphical art. It is hoped that this will be helpful to the recreation of busy conference participants at the $25^{\text {th }}$ World Congress and General Assembly of the International Union of Crystallography. Using some of Emil Makovicky's words from the second direct quote above, information theory based crystallographic symmetry classifications are poised not only to help resolve controversies in the symmetrology of art and cultural artifacts field [8], but also in the "natural sciences wherefrom the science of symmetry originated" [2]. 
[1] E. Makovicky, The crystallographic art of Hans Hinterreiter, Zeits. für Kristallogr. 150 (1979) 13-21.

[2] E. Makovicky, Symmetrology of art: coloured and generalized symmetries, Computers Math. Applic. 12B (1986) 949-980.

[3] E. Makovicky, Ornamental Brickwork, Theoretical and applied symmetrology and classification of patterns, Computers Math. Applic. 17 (1989) 955-999.

[4] E. Makovicky, Symmetry through the eyes of the old masters, Berlin: De Gruyter, 2016.

[5] E. Makovicky and M. Ghari, Neither simple nor perfect: from defect symmetries to conscious pattern variations in Islamic ornamental art, Symmetry: Culture and Science 29 (2018) 279-301.

[6] P. Moeck, Towards generalized noise-level dependent crystallographic symmetry classifications of more or less periodic crystal patterns, Symmetry 10, paper 133 (46 pages) (2018), doi: 10.3390/sym10050133, open access.

[7] P. Moeck, On classification approaches for crystallographic symmetries of noisy 2D periodic patterns, IEEE Trans. Nanotech. 18 (2019) 1166-1173, doi: 10.1109/TNANO. 2019.2946597, expanded version (21 pages) arXiv: 1902.04155v4, May 29, 2021.

[8] E. Makovicky, Comments on decagonal and quasi-crystalline tilings in medieval Islamic architecture, Science 318, Art. no. 1383a, (2 pages) 2007.

Keywords: Crystallographic Image Processing, Information Theory 